\title{
Response of the authors to the comments of Josef Josef Finsterer and Sinda Zarrouk-Mahjoub on Neurolmage "Cerebral arterial and venous MRI abnormalities in MELAS"
}

\author{
Dimitri Renard ${ }^{1} \cdot$ Ioana Ion ${ }^{1}$
}

Received: 5 June 2018 / Accepted: 7 June 2018 / Published online: 11 June 2018

(c) Belgian Neurological Society 2018

We would like to thank Josef Josef Finsterer and Sinda Zarrouk-Mahjoub for their excellent comments on our NeuroImage "Cerebral arterial and venous MRI abnormalities in MELAS".

Hereby, we will give some more clinical and radiological details on the three MELAS patients reported. All three patients had the classical m.3243A>G mutation. The first patient was a 32-year-old man with a history of seizures treated by topiramate $75 \mathrm{mg}$ /day presenting with a strokelike episode (SLE) in the absence of clinical or EEG abnormalities in favor of epilepsy. ADC map showed cortical hypointensity in the involved brain region in the absence of subcortical signal abnormalities. The SLE was treated by high-dose L-arginine in the acute phase. The second patient was a 20-year-old man presenting with involuntary unilateral lower limb movements evoking partial motor seizures. ADC map showed isointensity in the cortex associated with hyperintensity in the underlying subcortical involved brain regions. The patient refused EEG and left the hospital with levetiracetam $1000 \mathrm{mg}$ /day antiepileptic therapy without other specific SLE treatment. The third patient was a 54-year-old woman with a history of epilepsy treated with lacosamide $200 \mathrm{mg} /$ day presenting with SLE in the absence of clinical or EEG abnormalities in favor of epilepsy. ADC map showed mixed hyper/hypointensities in the cortex and hyperintensities in the underlying subcortical brain regions. The SLE was treated by high-dose L-arginine in the acute phase.

We agree with the comments of the author that the arterial abnormalities reported were probably not causative but rather a phenomenon secondary to the primary metabolic

Dimitri Renard

dimitrirenard@hotmail.com

1 Department of Neurology, Nîmes University Hospital, CHU Nîmes, Hôpital Caremeau, 4 Rue du Pr Debré, 30029 Nîmes Cedex 4, France defect. Concerning the possible role of seizures and/or SLE treatment in the arterial abnormalities reported, in our opinion, this seems unlikely, since two of the three patients lacked clinical and EEG abnormalities in favor of epilepsy, and arterial abnormalities were restricted to the brain areas involved by the SLE. One might suspect a more diffuse cerebral artery dilatation in treatment-induced perfusion abnormalities, although an interaction between SLE treatment therapies and cerebral arteries only involved in the SLE cannot be excluded.

The variability of signal changes on ADC map in SLE in our three patients corresponded well to the earlier reports analyzing ADC in SLE showing cortical hypo-, iso-, or hyperintensities during SLE, probably also depending on the timing of MRI performance (and the stage of SLE) [1, 2].

\section{Compliance with ethical standards}

Conflict of interest We have no conflict of interest to declare.

Ethical statement The manuscript was approved by the institute's committee on human research.

Informed consent The subject has given her informed consent to report data given.

\section{References}

1. Kim JH, Lim MK, Jeon TY et al (2011) Diffusion and perfusion characteristics of MELAS (mitochondrial myopathy, encephalopathy, lactic acidosis, and stroke-like episode) in thirteen patients. Kor J Radiol 12:15-24

2. Ito $\mathrm{H}$, Mori K, Harada $\mathrm{M}$ et al (2008) Serial brain imaging analysis of stroke-like episodes in MELAS. Brain Dev 30:483-488 